\title{
Promising early outcomes of surgery
}

The 12-week functional outcomes of robot-assisted laparoscopic prostatectomy and radical retropubic prostatectomy for newly diagnosed localized prostate cancer are similar, according to new data published in The Lancet. Both techniques have good early results, so patients should chose a surgeon they trust rather than a specific technique.

Yaxley et al. performed a phase III study involving men who had chosen a surgical treatment approach for their prostate cancer. Men were assigned 1:1 to receive either robot-assisted laparoscopic prostatectomy or radical retropubic prostatectomy. The primary outcomes were urinary function and sexual function. Overall, 12-week questionnaire data were available for 121 who underwent robot-assisted laparoscopic prostatectomy and 131 patients who received radical retropubic prostatectomy.

Urinary function and sexual function scores did not differ significantly between men who had received either surgical technique at the 6-week and 12-week postoperative time points. Equality between the two techniques could not be established based on a $90 \%$ confidence interval with a $\Delta$ of $10 \%$ using equivalence testing on the difference in the proportion of positive surgical margins (10\% in the radical retropubic prostatectomy group versus $15 \%$ in the robot-assisted laparoscopic prostatectomy group); however, superiority testing showed that the two proportions were not significantly different. Postoperative complications were experienced by $9 \%$ of men who underwent radical retropubic prostatectomy and $4 \%$ of patients who received robot-assisted laparoscopic prostatectomy, which was not significantly different.

This study is the first randomized trial comparing these prostatectomy approaches and no significant difference in functional outcomes was observed at the 12 -week postoperative time point. Longer-term follow-up data is required, but in the meantime patients should chose a surgeon they trust rather than a particular surgical approach. 